\title{
Antivirals become a broader enterprise
}

\author{
Jeffrey L Fox
}

Small molecules have dominated antivirals for decades, but other approaches are needed and biologics could show the way.

E fforts to develop antiviral therapeutics had mostly sputtered before the AIDS epidemic spurred an intensive search for new drugs to counter the human immunodeficiency virus (HIV). Added impetus has been provided by the frustrations and failures encountered during HIV vaccine development (Box 1). Although ideally vaccines are the way to go with this and other damaging and deadly viruses, the cumulative experience with HIV continues to prove that therapeutics are likely to remain a crucial part of the antiviral armamentarium.

Early explorations in antivirals focused mainly on small-molecule drugs for disrupting HIV and a handful of other viruses, notably influenza. A limited number of higher-molecular-weight entities (proteins) were also considered-most notably, interferon- $\alpha$ (IFN- $\alpha)$, which rose to prominence as a cure-all treatment for boosting antiviral immune responses. For the past two decades, however, small molecules have dominated the commercial sector, with combinations of nucleoside and nonnucleoside reverse transcriptase inhibitors or protease inhibitors against HIV—highly active anti-retroviral therapy (HAART) - becoming a multibillion-dollar annual enterprise.

But that scene is changing, as monoclonal antibodies (mAbs), nucleic acid therapies and other biologics are being tested in viral disease and the emphasis is broadening from virus targets to encompass targets within the host involved in immune responses or other virus-cell interactions (Fig. 1 and Tables 1 and 2). What's more, the traditional paradigm of narrow-spectrum, one drug-one virus products is being challenged. Some antiviral agents currently under development could almost be considered 'broad' (or at least 'medium') spectrum because they act against more than one type of virus. In some cases, this search depends on investigating broad but marginal antiviral

Jeffrey L. Fox is a contributing writer for Nature Biotechnology.

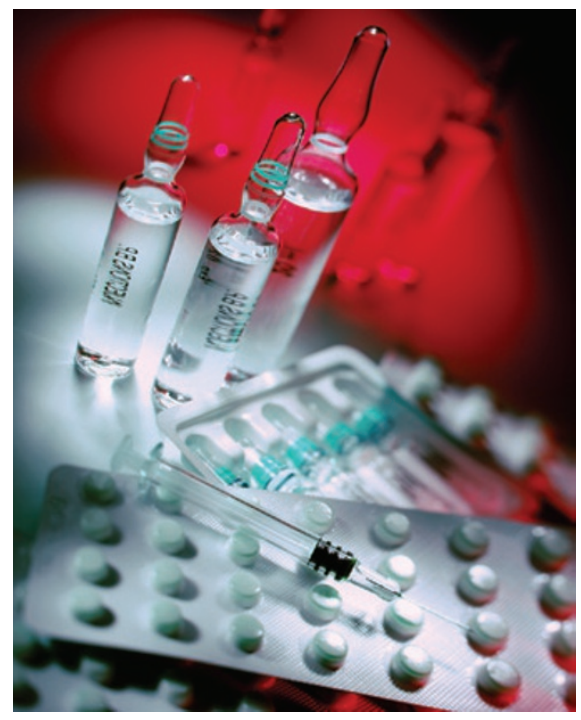

Antiviral therapeutics take various forms, but the contribution of biologics may be on the rise. Source: Newscom.com/Steve Allen

activity in drugs already being marketed for nonviral diseases such as cancer.

\section{Unmet needs}

HIV remains a major target for companies developing innovative antiviral treatments, some of which involve new approaches to blocking the entrance, maturation or departure of this virus into, within and from host cells (Fig. 2). Moreover, the AIDS epidemic continues to grow. Worldwide, about 40 million people are infected with HIV, and $>4$ million become infected each year, according to estimates from the World Health Organization (WHO; Geneva, Switzerland). In the United States, more than 1 million are infected with HIV, and roughly 40,000 new infections are added each year, according to estimates from the US Centers for Disease Control and Prevention (CDC; Atlanta, GA).

By sheer numbers of infected individuals, hepatitis $\mathrm{C}$ virus ( $\mathrm{HCV}$ ) is also a major health problem. According to $\mathrm{WHO}$, anywhere from 100-200 million people are infected with HCV and hence are at risk for developing cirrhosis and liver cancer. The challenge of treating HCVinfected individuals is compounded by the absence of a preventive vaccine, the long-term consequences of the infection for patient quality of life and the poor response rate to existing standard therapy - a combination of IFN- $\alpha$ and ribavirin. According to Rahul Jasuja, vice president for research at MDB Capital Group (MDB; Santa Monica, CA, USA), the development of new therapeutic agents for HCV is "critical"; he notes that only $\sim 60 \%$ of such patients respond to combination treatment, particularly those infected with HCV genotype 1.

Similarly, although a vaccine is helping to control hepatitis B virus (HBV), WHO officials estimate that two billion people are infected with HBV globally and that more than 350 million have chronic infections with this virus, whose long-term consequences are similar to those of HCV. (HCV accounts for about two-thirds of $\$ 1.6$ billion in sales for IFN- $\alpha$, with the other one-third going to treat HBV infections and cancer.) Even in the United States, over 1 million people are estimated to be carriers of $\mathrm{HBV}$.

Another virus responsible for a widespread chronic infection is human papilloma virus (HPV), which leads to $\sim 500,000$ new cases of cervical cancer each year and 232,000 deaths worldwide. But here, at least, there is some good news; in June 2006, the US Food and Drug Administration (FDA; Rockville, MD) approved Merck's (Whitehouse Station, NJ) HPV vaccine Gardasil, which protects uninfected individuals against HPV subtypes 6/11/16/18. By all accounts, the vaccine has been a remarkable commercial success, with sales over a billion dollars.

Although the numbers of infections caused by other viruses are far lower, they are still being targeted by companies. In particular, commercial interest has recently focused on emerging 
pathogens, such as West Nile virus (WNV), severe acute respiratory syndrome (SARS) coronavirus and $\mathrm{H} 5 \mathrm{~N} 1$ influenza virus.

Despite continuing difficulties surrounding government grants, some firms are also addressing potential bioterror agents, such as Ebola, Marburg and smallpox viruses (Box 2). Vaccinia is a special case. Used in live form as a vaccine to protect against smallpox-a disease that no longer occurs in its natural form-it can sometimes cause harmful infections among those (mainly those serving in the military) who are vaccinated against smallpox and others with whom they come in contact, particularly if they are immunocompromised. Thus, new treatments are actively being sought.

\section{Small molecules predominate}

A glance at the third-quarter report from Gilead Sciences (Foster City, CA) helps to explain why there is such widespread interest among biotech companies in antiviral product development. Gilead, which calls itself a 'biopharmaceutical company,' reports record sales of just under \$962 million for the third quarter, noting that this growth "was driven primarily by [its] HIV product franchise.” Sales for HIV products were about $\$ 806$ million for the third quarter, representing the lion's share of overall sales revenue, whereas sales of Hepsera (adefovir dipivoxil), a nucleotide analog used to treat chronic HBV, accounted for $\$ 79$ million during that period. Major pharmaceutical companies are seeing similar growth in sales of drugs for treating HIV infections. For example, Bristol-Myers Squibb (Princeton, NJ, USA) reports $\$ 273$ million in worldwide sales of its HIV protease inhibitor Reyataz (atazanavir sulfate), representing $17 \%$ growth for the third quarter compared with 2006. Although the overall market size is much smaller for diagnostics than it is for drugs, viral diagnostics garnered by far the largest share of the in vitro diagnostics market, which in 2006 took in over $\$ 1.5$ billion and is expected to do so going forward (Box 3 and Fig. 3 ).

Regardless of these sales figures, however, HIV's high mutation frequency, which leads rapidly to development of resistance to such drugs, keeps a fire under corporate antiviral therapeutic development programs. It also sparks developments leading toward unconventional products-namely, those that do not simply duplicate the actions of small-molecular-weight molecules with the same proven vulnerabilities reflected in anti-HIV drugs currently on the market. This is not to say that there is no longer interest in developing nucleoside and non-nucleoside inhibitors for the HIV reverse transcriptase as well as inhibitors for the HIV protease (Huddinge, Sweden-based Medivir's small molecule reverse transcriptase inhibitor MIV-210 is a case in point); but the strategies are changing.

Many academic and biotech discovery programs are looking to new therapeutic

\section{Box 1 The struggle for an HIV vaccine}

In part because HIV targets and undermines the immune system and in part because of its rapid capacity to mutate its antigens, many ingenious efforts to develop protective vaccines against it have proved fruitless. Just this past September, for example, officials at NIAID and Merck called a halt to two clinical trials to evaluate Merck's V520 HIV vaccine (a trivalent vaccine of gag, pol and nef, one in South Africa and the other at sites in Australia, Brazil, Canada, the Dominican Republic, Haiti, Jamaica, Peru, Puerto Rico and the United States. An independent Data Safety Monitoring Board had concluded that the V520 "could neither prevent HIV infection nor reduce the amount of virus in those who became infected with HIV." Moreover, volunteers who participated in this study, referred to as the "Phambili," HVTN 503 in South Africa or HVTN 502 or STEP at the other sites, are also being counseled about the possibility that vaccine recipients "may have an increased susceptibility to acquiring HIV infections."

Against such a backdrop, it is "not immediately obvious why an immunomodulatory product is suitable for HIV-infected, immunocompromised patients," says Charles Nicolette, vice president for R\&D of Argos Therapeutics (Durham, NC, USA). Moreover, according to his review of the recent scientific literature, "immunotherapy has a complete lack of efficacy in terms of controlling the viral load among HIV-infected patients, even in cases where immunity is augmented," he adds, alluding to dozens of published reports. "The story is the same in every case."

"Maybe they were all looking at the wrong antigens," Nicolette continues. Instead of looking for a single antigen, Argos goes after HIV-infected individuals, extracts a medley of viral antigens from each person, reverse engineers and amplifies by PCR messenger RNA (mRNA) molecules corresponding to those viral antigens, and then uses electroporation to load that mRNA into dendritic cells. The cells are introduced into each patient to induce an individually tailored immune response to whatever "quasi-species" of HIV that he or she is carrying, according to Nicolette. Following phase 1 studies, which uncovered no unusual safety problems, plans call for administering such cocktails early in 2008 to HIV-infected individuals who are taking antiviral drugs and then measuring viral loads when drug therapy is temporarily halted, he says. "We expect to have a full data set by the fourth quarter of 2009."

Other HIV-immunomodulatory products under development are closer to being conventional vaccines-at least, in the sense that products are not being separately tailored to suit each infected individual. Among the "dozens of designs" being followed and evaluated, Wyeth (Madison, NY, USA) researchers are looking at a DNA-based HIV-therapeutic vaccine that consists of two plasmids, one encoding HIV immunogens and the other encoding interleukin-12 (IL-12) to act as an adjuvant, according to John Eldridge, vice president of applied vaccines research at Wyeth (Pearl River, NY). The underlying idea is to use therapeutic vaccines with antiviral drugs to "bring the immune system back up and maybe halt antiretrovirals if the immune system can control the virus and reduce damage," he says.

Elsewhere, The Vaccine Research Center at the National Institutes of Health (NIH; Bethesda, MD) is testing a 'prime/ boost' vaccine incorporating the plasmid DNA technology of Vical (San Diego, CA) to prime the immune system and the adenoviral vector (Ad35) technology of GenVec (Gaithersburg, MD) as an immune booster. A phase 1 trial with both a single-dose arm and a dose-escalation arm began in June.

Among the several companies developing therapeutic vaccines for HIV, "it's difficult to say somebody is ahead," Eldridge says. "Many people are working with very difficult targets." Although at least one of those other vaccines is in phase 1 clinical trials, plans call for evaluating the Wyeth vaccine in rhesus macaques in a one-year study that has yet to begin. "Although it's difficult to extrapolate from [the STEP prophylactic vaccine trial in South Africa] to a therapeutic, that trial gives us pause, and we're not sure what's going on or what to predict," he says. Still, none of the animal studies involving those therapeutics "predict any damage." 
modalities. "We don't have much to say about small-mole-cule inhibitors of HIV," says Sandra Bridges at the National Institute of Allergy and Infectious Diseases (NIAID) at the National Institutes of Health (NIH; Bethesda, MD). Soon after HIV was identified, the federal government launched programs to encourage the development of such drugs, and the avid involvement $>$ of the biotech and pharmaceutical industries led the way through nearly two decades of arduous research and development (R\&D).

Today, small-molecule drug development is "largely in the hands of the pharmaceutical industry," Bridges continues. "Our recent focus is on nontraditional approaches." Some of that nontraditional development is coming squarely from biotech companies, whereas another portion depends on collaborative arrangements with researchers at universities.

One innovative compound that came out of a group at Duke University (Durham, NC), was licensed by Trimeris (Morrisville, NC) and was finally co-developed with Roche (Basel, Switzerland) is Fuzeon (enfuvirtide; T20). 윽 Fuzeon, which was approved by the FDA in
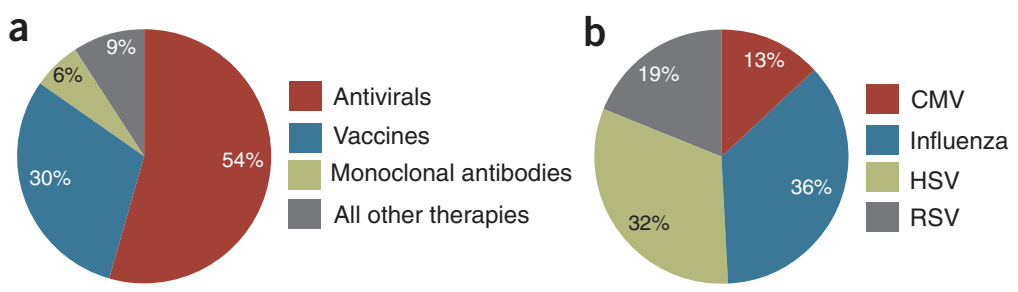

Figure 1 Antiviral therapeutics market. (a) Antivirals garner the largest share of the market (54\%, $\$ 18$ billion), with vaccines (30\%, $\$ 19$ billion) and mAbs (6\%, \$2 billion) getting smaller shares. (Source: Jain KK. Antiviral Therapeutics. Jain PharmaBiotech Publications, Basel, November 2007.) (b) 2006 US market share of anti-viral agents by indication (excluding HIV). (Source: Arrowhead Publications, Minneapolis, MN.)

2003, is an injectable synthetic peptide derived from the C-terminal (heptad repeat sequence 2, HR2) domain of gp41. By binding amino acids 643-678 in HIV-1 gp41, Fuzeon interferes with the fusion of HIV with $\mathrm{CD} 4^{+}$cells. Unfortunately, the drug has had a difficult time commercially. This has been attributed to the inconvenience of injections (as compared with oral administration of small-molecule inhibitors) and the drug's propensity to cause welts at the injection site.
In the meantime, small molecules have been screened against new HIV targets in an attempt to intervene at new points in the virus life cycle. For example, last October the FDA approved Merck's Isentress (raltegravir), the first in the class of HIV integrase inhibitors, for clinical use in combination with other antiretroviral agents.

Bevirimat, which is being developed at Panacos Pharmaceuticals (Watertown, MA, USA)-yet another first-in-class antiviral targeting HIV-interferes with late-stage

Table 1 Selected antivirals in clinical trials

\begin{tabular}{|c|c|c|c|}
\hline Company & Antiviral approach & Target viruses & Clinical trial status \\
\hline Alnylam (Cambridge, MA) & RNAi & Influenza, RSV & Phase 2 \\
\hline Argos (Durham, NC) & Personalized immunotherapy & HIV & Phase $1 / 2$ \\
\hline AVI Biopharma (Portland, OR) & Antisense (Neugene) & $\mathrm{HCV}$ & Phase $1 / 2$ \\
\hline Benitec Melbourne, Australia & Cell-based therapy/triple RNAi & HIV & Phase 1 \\
\hline CytoDyn (Santa Fe, NM) & $\mathrm{mAb}$ & HIV & Phase $1 / 2$ \\
\hline Crucell (Leiden, the Netherlands) & Antibody cocktail & Rabies & Phase 1 \\
\hline Dynavax (Berkeley, CA) & TLR9 agonist (Heplisav) & HBV & Phase 3 \\
\hline Enzo Therapeutics (New York, NY) & Antisense gene therapy & HIV & Phase $1 / 2$ \\
\hline Human Genome Sciences (Rockville, MD) & $\begin{array}{l}\text { Modified interferon (Albuferon); mAb blocks } \\
\text { CCR5 }\end{array}$ & $\begin{array}{l}\text { HCV } \\
\text { HIV }\end{array}$ & $\begin{array}{l}\text { Phase } 3 \\
\text { Phase } 1\end{array}$ \\
\hline Idera (Cambridge MA) & Oligonucleotide-based TLR9 agonist & $\mathrm{HCV}$ & Phase 1 \\
\hline $\begin{array}{l}\text { Implicit Bioscience (Seattle, WA; Brisbane, } \\
\text { Australia) }\end{array}$ & Peptide immunomodulators & HCV, influenza & Phase 2 \\
\hline MacroGenics (Rockville, MD) & mAb blocks CCR5 & West Nile virus & Phase 1 \\
\hline MedImmune (Rockville, MD) & mAb (Numax, optimized Synagis) & RSV & $\begin{array}{l}\text { Phase } 3 \text { completed (high-risk children); } \\
\text { phase } 1 \text { (adults) }\end{array}$ \\
\hline NexBio (San Diego, CA) & Recombinant sialidase & Influenza & Phase 1 \\
\hline Nucleonics (Horsham, PA) & RNAi & HBV & Phase 1 \\
\hline Panacos (Watertown, MA) & Maturation inhibitors (bevirimat) & HIV & Phase 2 \\
\hline Polymun Scientific (Vienna, Austria) & mAb cocktail & HIV & Phase 2 \\
\hline Progenics (Tarrytown, NY) & mAb blocks CCR5 & HIV & Phase $1 b$ \\
\hline Protiva (Burnaby, BC, Canada) & RNAi & Ebola, Marburg & Phase 1 \\
\hline Romark Laboratories (Tampa, FL) & Mechanism unknown (nitazoxanide) & $\mathrm{HCV}$ & Phase 2 \\
\hline Schering-Plough (Kenilworth, NJ) & Protease inhibitor (boceprevir) & $\mathrm{HCV}$ & Phase 2 \\
\hline SciClone Pharmaceuticals (Foster City, CA) & Immunomodulator (Zadaxin, thymalfasin) & HBV, HCV & Marketed (HBV); phase 3 (HCV) \\
\hline SIGA Technologies (New York, NY) & Viral particle blocker & Smallpox, other orthopox & Phase 1 \\
\hline United Biomedical (Hauppage, NY) & Immunotherapeutic peptides & HIV & Phase 1 \\
\hline Vertex Pharmaceuticals (Cambridge, MA) & Protease inhibitor (telaprevir) & $\mathrm{HCV}$ & Phase 2 \\
\hline VGX Pharmaceuticals (Blue Bell, PA) & Steroid abortifacient (mifepristone) & HIV, HCV & Phase 2 \\
\hline VIRxSYS (Gaithersburg, MD) & Gene-based immunotherapy & HIV & Phase 1 \\
\hline
\end{tabular}


Table 2 Selected companies with antivirals in preclinical development

\begin{tabular}{lll}
\hline Company & Antiviral approach & Target virus \\
\hline AVI Biopharma (Portland, OR) & Antisense & Ebola, Marburg \\
\hline CytoDyn (Sante Fe, NM) & DNA vaccine & Influenza \\
\hline Functional Genetics (Gaithersburg, MD) & mAb targets 'hijacked' cell protein (TSG101) & Multiple targets \\
\hline Isis Pharmaceuticals (Carlsbad, CA) & Oligonucleotide aptamer & HIV \\
\hline Presidio (San Francisco, CA) & Viral genome nuclear import blocker & HIV \\
\hline Regulus (Carlsbad, CA) & microRNA & HCV, others \\
\hline REPLICor (Laval, Quebec, Canada) & Amphipathic DNA polymers & HCV \\
\hline Sangamo BioSciences (Richmond, CA) & Zinc-finger nucleases & CCR5 receptor/HIV \\
\hline SIGA Technologies (New York, NY) & Viral particle blocker & Lassa \\
\hline Symphogen (Lyngby, Denmark) & Polyclonal antibodies & RSV, vaccinia \\
\hline Tacere Therapeutic (San Jose, CA) & Short hairpin RNA (shRNA) & HCV \\
\hline
\end{tabular}

maturation of the viral gag protein, allowing the release of particles that appear intact but are noninfectious. This low-molecular-weight derivative of betulinic acid is "providing very positive results in phase 2 clinical testing," says Panacos COO Graham Allaway, adding that bevirimat has "good oral availability [and] long half-life, is well-tolerated, and is metabolized differently from other antiretroviral drugs." As yet, no other companies appear to be working on similar maturation inhibitors.

Several companies with small-molecule programs are also using HIV to provide clues to druggable targets in other viruses. For example, similar to HIV protease, the HCV NS3-4A serine protease has long been thought to be a promising target for antivirals. At the annual meeting of the American Association of the Study of Liver Diseases last month, Vertex Pharmaceuticals (Cambridge, MA), announced that its small molecule telaprevir (VX-950), when used with standard combination therapy of ribavirin and PEGylated IFN- $\alpha$, induced viral response rates of $>61 \%$ in one phase $2 b$ trial and 65\% in another. At the same meeting, Schering-Plough (Kenilworth, NJ) announced even more positive results from a phase 2 trial for its protease inhibitor boceprevir.

Meanwhile, Romark Laboratories (Tampa, FL, USA) reported that the results of a phase 2 clinical trial of its small molecule Alinia (nitazoxanide) in HCV-infected patients showed a $79 \%$ sustained virologic response. Nitazoxanide was initially developed as a treatment for cryptosporidiosis, a parasitic disease, and its mechanism of action is still unclear. Yet another target, HCV polymerase, has also been singled out as a promising focus for antivirals, but this field has suffered several clinical setbacks in the past few months (see p.1379).

\section{Nucleic acid therapies emerge}

If any biotech company is synonymous with the rise of RNA interference (RNAi), it would be Cambridge, Massachusetts-based Alnylam Pharmaceuticals. It is particularly significant, therefore, that the firm's first lead product is an antiviral.

According to COO Barry Greene, ALNRSV01, a small interfering RNA (siRNA) that targets the mRNA for the nucleocapsid protein of respiratory syncytial virus (RSV), is now being evaluated in a phase 1 inhalation study, and results are expected soon. The company is also developing a human experimental infection model for RSV, which ordinarily infects children or the elderly, but here is being developed for testing in adult volunteers. There is "no effective treatment for RSV infection," he says, noting that $65 \%$ of children become infected with RSV during the first year of life, and more than 170,000 elderly are hospitalized with it annually, leading to more than 14,000 deaths.
Although MedImmune (Gaithersburg, MD, USA; now a subsidiary of AstraZeneca) already markets human mAb Synagis (palivizumab) against RSV, it is used only in the prophylactic treatment of prematurely born infants.

Another RNAi developer is Nucleonics (Horsham, PA, USA). Last May, the company received FDA clearance to begin phase 1 testing of its siRNA therapy for treating chronic HBV infections. Its approach entails inserting plasmid DNA encoding short hairpin RNA (shRNA) molecules that can silence viral (or other) genes. In this, the plasmid carries four such sequences targeting different segments of the HBV genome, interfering with the RNA molecules that the virus produces during its replication cycle.

Another young company, Regulus Therapeutics (Carlsbad, CA), is applying microRNA antisense technology to HCV therapeutics, according to CSO Frank Bennett. This initial focus on HCV, despite "a lot of competition," comes in part because this medical need is still "underserved." However, it is also because the candidate products being developed naturally gravitate to the liver (in rodents, at least) and not only disrupt HCV but also lower cholesterol levels and reverse other pathologies there, he says. "We are taking advantage of that natural biodistribution... to get very good drug levels in the liver without sophisticated formulations." The company, which was launched in September as a joint venture of Alnylam and Isis Pharmaceuticals (Carlsbad, CA), anticipates starting phase 1 clinical trials by late 2008 or early 2009.

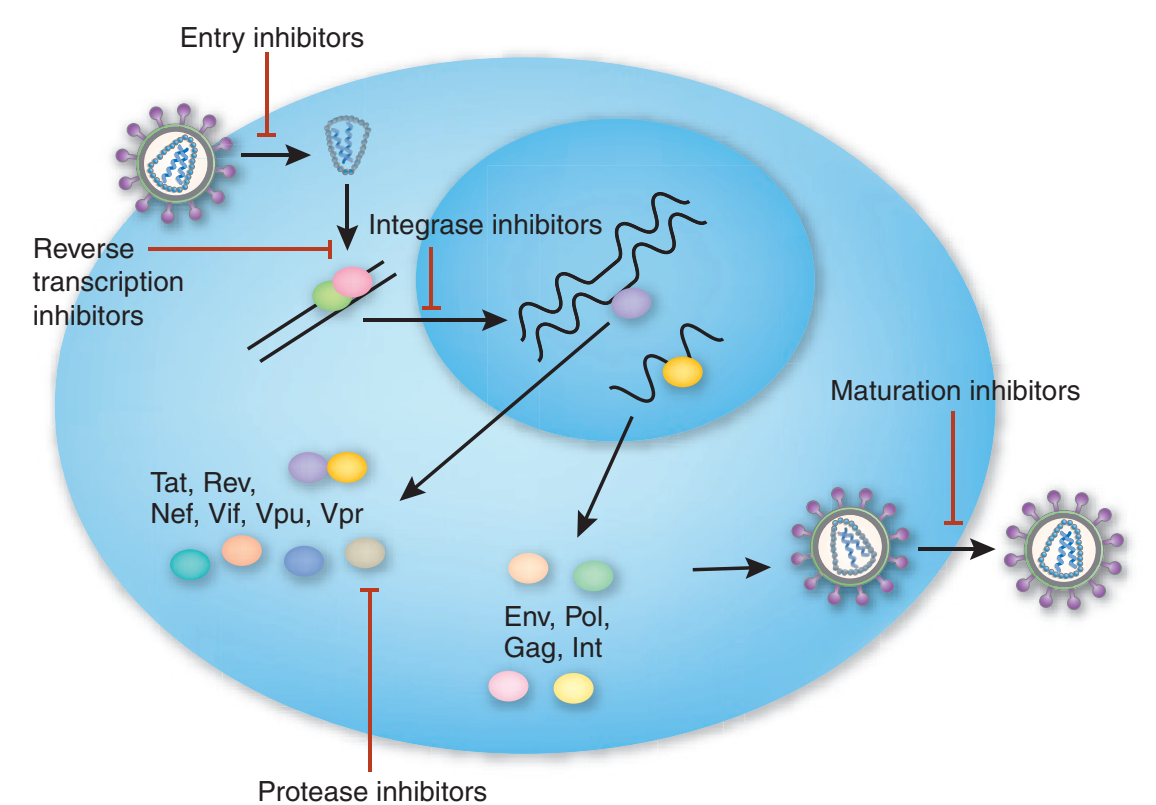

Figure 2 HIV live cycle and points of intervention by antiviral drugs. 
In Laval, QC, Canada, Replicor is developing a rather different type of nucleic acid molecule. Its amphipathic, 40-phosphodeoxynucleotidecontaining molecules target conserved $\alpha$-helical domains of envelope proteins found among a variety of viruses. According to CSO Andrew Vaillant, the company is focusing initially on $\mathrm{HCV}$ and HBV."These compounds are well tolerated in mice and nonhuman primates... and are highly amenable for treating liver disease," he says. "Targeting is independent of the [target protein's] amino acid sequence, so point mutations won't confer resistance." Vaillant suggests Replicor's candidate antiviral drugs could begin clinical testing by the end of 2008 .

AVI Biopharma is yet another drug maker focusing on developing antisense molecules against viral pathogens. In July, it presented preclinical data at the 26th Annual Meeting of the American Society for Virology in Corvallis, Oregon showing that its antisense morpholino oligomers (NeuGene) inhibited measles virus replication and titer by two logs in plaque assays and showed efficacy against murine hepatitis virus and Venezuelan equine encephalomyelitis virus in mouse models.

VIRxSYS (Gaithersburg, MD, USA) is also pushing forward with an antisense treatment involving a negative-strand sequence carried on a vector based on a lentivirus (a modified version of HIV); this is being administered $e x$ vivo to $\mathrm{T}$ cells of patients in phase 2 clinical tests, according to Gerard McGarrity, who is vice president for scientific and clinical affairs, and Laurent Humeau, vice president for R\&D. Results from 2006 phase 1 studies involving a very limited set of patients were "encouraging, showing decreases in viral loads, even among patients on drug therapy for many years," McGarrity says. Phase 2 findings are expected early in 2008.

The sequence segment in VRX496 comprises 937 nucleotide bases that target the viral gene encoding its envelope protein, following a rationale that this long target makes it difficult for even a "hypermutating HIV to mutate around it," McGarrity says. With HIV, “we never say never, but it will be extremely difficult in vitro to find a way around this. We also think this approach circumvents the toxicities that HIVinfected patients face."

Similarly, Enzo Therapeutics (New York, NY) launched a phase $1 / 2$ clinical trial early in 2007 , transferring antisense genes directed against viral genes required for replication (the tar gene and two sites in the tat/rev gene) via its Moloney murine leukemia virus-derived vector HGTV43 into T cells of HIV-infected individuals. This 'personalized' approach to vector-borne antisense therapy also entails a step in which each patient is irradiated to reduce resident stem cells before the exvivo-treated, genetically engineered $\mathrm{T}$ cells are reinfused.

\section{Monoclonals mount a response}

Not surprisingly, given the pre-eminence of these agents in other therapeutic areas, several companies are turning their attention to the development of antibody therapeutics - mainly

\section{Box 2 Bioterror-inspired approaches}

Still other viruses are attracting attention, much of it involving conventional approaches to drug development, but some of it slightly offbeat. Perhaps some of those unconventional approaches can be attributed to lingering influence from the Defense Advanced Research Projects Agency (DARPA; Arlington, VA), which is no longer working on antivirals, according to a spokesperson. However, the Defense Threat Reduction Agency (DTRA; Ft. Belvoir and Alexandria, VA) continues to issue contracts supporting biotech companies that work on antiviral products. Moreover, the NIH supports antiviral product development in the private sector, particularly through the Small Business Innovation Research (SBIR) program.

For example, last August DTRA awarded Alnylam (Cambridge, MA, USA) a \$38.6 million contract to develop broad-spectrum antiviral products based on its RNA interference (RNAi) approach, for diseases including viral hemorrhagic fever. NIH awarded the company a $\$ 23$ million contract in 2006 to develop therapeutic products for the Ebola virus. Similarly, AVI BioPharma (Portland, OR, USA) has received a series of multimillion-dollar contracts from the Department of Defense, including some from DTRA, to develop morpholino antisense (NeuGene) therapeutics against such pathogens as Ebola and Marburg viruses. In preclinical testing, one candidate product protected nonhuman primates against Ebola virus infections.

Separately, Protiva Biotherapeutics (Burnaby, BC, Canada) is developing short interfering RNA (siRNA) technology aimed at several viruses of biodefense interest, such as Ebola, and other viruses such as influenza, HBV and HCV for general use, according to president and CEO Mark Murray. "Our particular angle is a very infective intracellular delivery vehicle for siRNA, whose efficacy critically depends on delivery," he says, referring to the company's stable nucleic acid lipid particle (SNALP) technology, which provides stability and extended serum half-lives for encapsulated siRNA molecules and was licensed by Merck in October. "In the Ebola case, this technology is extraordinarily effective," he says. "Animals survive otherwise lethal infections, and their serum is aviremic 14 days later."

Siga Technologies (New York, NY, USA) is developing several candidate small-molecule drugs targeting several viruses of biodefense interest, including smallpox and other orthopox viruses as well as Lassa fever virus. In September, the company reported that its ST-246, its leading small-molecule candidate against orthopox, protected $100 \%$ of cynomolgus monkeys against a lethal dose of monkeypox virus in tests conducted at the US Army Medical Research Institute of Infectious Diseases (USAMRIID; Frederick, MD) facilities. Monkeypox virus is closely related to variola, the virus that causes smallpox in humans. Earlier in March, under an emergency investigational new drug designation granted by the FDA, administration of ST246 to a child who had developed eczema vaccinatum after receiving live vaccinia vaccine, resulted in a complete resolution of the child's symptoms. In a separate study at USAMRIID that was reported last May, low doses of Siga's ST-193 antiviral candidate product protected $71 \%$ of guinea pigs against an otherwise lethal dose of Lassa fever virus.

Other companies, including MacroGenics (Rockville, MD, USA) and Symphogen (Lyngby, Denmark), are developing both $\mathrm{mAbs}$ and polyclonal antibodies for possible use in neutralizing smallpox or other orthopox viruses, including vaccinia, which is used to vaccinate active military and other individuals to protect them against smallpox. Although safe for most people, vaccinia sometimes causes a disseminated infection that now is treated with a mix of immunoglobulins. "Our mAb cocktail is much more potent when tested in mice," says Jeffrey Nordstrom, director of product development at MacroGenics. Meanwhile, Symphogen is evaluating recombinant polyclonal antibodies for several applications, including against disseminated vaccinia and smallpox infections and those caused by RSV, both of which are in preclinical development. "In our RSV program we have found that antibody mixtures are significantly more potent than when tested individually," says John Haurum, Chief Scientific Officer at Symphogen. 
mAbs, but also polyclonals - to combat a variety of viral infections. Last July, the FDA approved MacroGenics' plans for a phase 1 clinical trial of its humanized mAb MGAWN1 that targets the envelope protein of West Nile virus (WNV). A year earlier NIH awarded the company $\$ 50$ million to develop MGAWN1, particularly to use in treating WNV-infected patients who develop symptoms involving the central nervous system (CNS).

"We were surprised to find that [MGAWN1] goes into the CNS when it's administered peripherally," says director of product devel- opment Jeffrey Nordstrom. "It's a small amount, but it's highly potent and may be enough to neutralize the virus." Current monitoring picks up about 5,000 WNV-infected individuals in North America each year, with many thousands of asymptomatic infections going undetected. At least one-third of the confirmed cases involve the CNS, and some individuals develop flaccid paralysis affecting the peripheral nervous system that resembles polio. The company is also developing $\mathrm{mAb}$ cocktails to work before and after infections caused by the avian influenza virus.
Crucell (Leiden, The Netherlands) is developing a cocktail of two human mAbs (CR-57 and CR-4098) to treat infections caused by rabies, another virus that affects the CNS but whose infections are deadly unless treated promptly before symptoms appear. In a phase 1 clinical trial, results of which were reported last October, healthy volunteers had neutralizing antibody (seroconverted) whether they received only the mAb cocktail or the cocktail along with a standard rabies vaccine, indicating that the two can be safely co-administered. Worldwide supplies of the rabies vaccine are considered

\section{Box 3 Infectious diseases driving the diagnostics bus}

The infectious disease market and in vitro diagnostics (IVD) in general are expected to grow steadily (Fig. 4). The need for more rapid diagnosis and the emergence of resistance in big markets like HIV and HCV are fueling the development engine. While the big diagnostics companies-Roche, Abbott and Becton Dickinson-duke it out with competing HIV viral load assays along with the traditional infection assays, small biotechs are garnering some attention for their innovative contributions to viral diagnosis.

Such companies are already reaping rewards in the form of deals with large pharma. Earlier this year, Monogram Biosciences (S. San Francisco, CA), which has a cadre of HIV-related assays, had its longstanding relationship with Pfizer expanded to include its tropism assay, which Pfizer used during the development of its co-receptor antagonist Selzentry (maraviroc) - a deal estimated to be worth $\$ 25$ million to the smaller company. Applying Monogram's Trofile assay in a pivotal phase 3 clinical trial to the selection of patients that expressed the CCR5 receptor on $\mathrm{CD} 4^{+} \mathrm{T}$ cells proved critical to the success of that trial. Patients expressing the CXCR4 receptor were considered unlikely to respond and hence were excluded from the trial. The FDA approved Selzentry for CCR5-positive HIV patients in August of this year; Monogram has since marketed the Trofile assay, which works by amplifying HIV RNA from patient samples, packaging it into particles and testing for infectivity on CCR5- and CXCR4-expressing cell lines.

Monogram reports alliances with a number of other pharmaceutical giants, including Merck, which reportedly used Monogram's PhenoSense assay in selecting a background antiretroviral treatment for testing its integrase inhibitor drug raltegravir. The Phenosense assay determines drug resistance in virus isolated from patients' blood.

Addressing a large market, HPV in women-made larger by the introduction of the HPV vaccine in 2006, which raised awareness of the issue-Digene (Gaithersburg, MD) has parlayed its hybrid capture test for the 13 subtypes of HPV associated with cervical cancer into a sweet acquisition deal. After enjoying several years of increasing sales since its first HPV diagnostic launch in 2000 , the company was acquired (in July 2007) by the giant diagnostics Qiagen for $\$ 1.6$ billion.
Some see the present situation with anti-virals as revolutionary. Bruce Patterson, a professor of pathology at Stanford Medical School and founder of the diagnostics company Invirion (Oak Brooke, IL), likens today's climate in virology to the way bacteriology flourished after the discovery of penicillin. Once there are therapies, it becomes necessary to identify the cause of a viral infection and to have ways of measuring drug susceptibility and resistance if it occurs, he points out. This is driving the growth rate and rate of change in viral diagnostics market, but even so, drug developers are out ahead of diagnostics. Invirion, which has a cell-based technology based on work from Patterson's laboratory, is working with companies early in development-it has a number of products going through phase 2 and phase 3 clinical trials - so that once a product is approved, the diagnostic will be in the ready

But the sector has its challenges. With new technologies come considerable barriers to adoption, as some newer techniques require expensive and difficult-to-operate instrumentation and are also likely to encounter physician resistance and lack of experience in applying tests appropriately and interpreting test results. Test developers have to tailor their products to the marketplace to achieve success. According to a 2006 Frost and Sullivan report, US Infectious Disease Diagnostics Market, this requires that US clinical laboratories develop "custom solutions" that take into account challenges such as testing volume, reimbursement rates in different locales and the focuses of physicians and clinical laboratories.

Laura Defrancesco

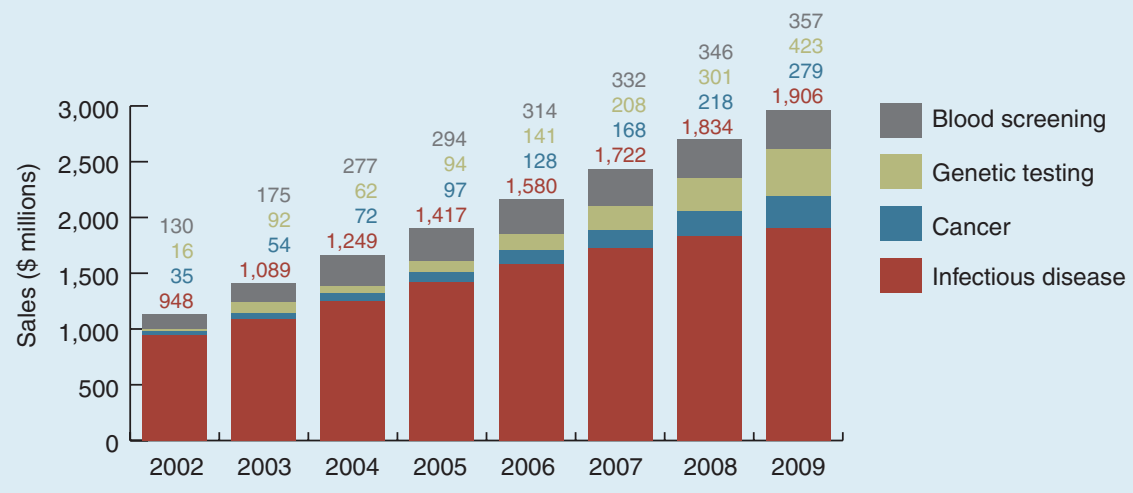

Figure 3 Market growth of in vitro diagnostics by sector from 2002-2006 with projections for 20072009. (Source: The BBI Newsletter, ACH Media Publisher.) 
adequate to treat those who are exposed to the rabies virus, which causes as many as 70,000 deaths per year, mainly in Asia. However, local vaccine shortages, particularly for Asia or other developing countries, and safety concerns about the immunoglobulin that is coadministered with it, present an opportunity to develop alternative treatments, according to Crucell. It forecasts annual sales exceeding $\$ 300$ million for its $\mathrm{mAb}$ immunoglobulin-replacement product. Crucell is also carrying out preclinical studies of several human mAbs, expressed using its PER.C6 cell line, for the potential treatment and prevention of $\mathrm{H} 5 \mathrm{~N} 1$ avian influenza virus infection.

Yet another biotech, Valley Cottage, New York-based XTL Biopharmaceutical has taken a combination of two fully human mAbs (Ab68 and Ab65) through phase 1 trials in HIV patients. In March, it announced the cocktail was well tolerated, although no reduction in viral load of HCV patients was observed.

Going one step futher, Polymun Scientific Immunbiogische Forschung (Vienna, Austria) is developing three human mAbs (2G12, 2FE and $4 \mathrm{E} 10)$ to be used together as a cocktail therapy against HIV. The $2 \mathrm{G} 12 \mathrm{mAb}$ recognizes a conserved dense cluster of oligomannose molecules on HIV's gp120 protein, whereas 2F5 and 4E10 bind to the membrane-proximal external region of HIV's gp41. The resultant effect is to block gp120 binding of the host CCR5 (C-C-motif receptor 5) co-receptor and gp41 binding with the host CD4 receptor. In a trial of eight chronically HIV-1-infected individuals undergoing interruption of antiretroviral treatment, analysis of 'escape' mutants - mutated forms of the virus that are no longer detectable by an individual's immune system - revealed that 2G12 was crucial for the neutralizing effect of the antibody cocktail. The clinical potency of these mAbs is currently being investigated in phase 2 trials.

Overall, the success of mAbs in breaching the small molecule-dominated antiviral market is likely to depend on whether their drawbacks (the inconvenience of injections, the difficulty of manufacture, their restricted penetration into tissue and their inability to block a spreading infection among resting cells) are outweighed by their strengths (a good safety profile and the lack of competition from generic products).

\section{Blocking host cell surface receptors}

Another major shift in antiviral development in recent years has been an increased emphasis of discovery programs on targeting host proteins rather than proteins specifically from the viral pathogens. One of the most obvious points for intervention in the virus-host interaction is host cell surface receptors. In August, this approach received its first proof of principle with FDA approval of Pfizer's (New York, NY) first-in-class antiretroviral product, Selzentry (maraviroc) - a small-molecule antagonist of the CCR5 co-receptor.

Selzentry is not alone-a veritable slew of molecules targeting CCR5 are now making their way through the clinic trials. For example, Incyte (Palo Alto, CA) announced in July that its small-molecule CCR5 antagonist INCB-9471 produced a mean $0.81 \log 10$ reduction in viral load and was well tolerated in a phase $2 \mathrm{a}$ trial.

Progenics' (Tarrytown, NY, USA) Pro140 is a humanized $\mathrm{mAb}$ being developed against HIV1 and HIV-2. In September, the company presented preclinical data at the 47 th Interscience Conference on Antimicrobial Agents and Chemotherapy in Chicago, Illinois, demonstrating potent in vitro activity (median $\mathrm{IC}_{50}$ $<0.2 \mu \mathrm{g} / \mathrm{ml}$ ) of Pro140 against HIV; interestingly, HIV escape mutants of Pro140 remain susceptible to another small-molecule CCR5 inhibitor, Schering-Plough's vicriviroc, suggesting that the two drugs act at different sites on the co-receptor. "Our mAb has great specificity and, unlike the Pfizer CCR5 blocker [maraviroc, which carries a black-box warning], no liver toxicity," claims Progenics CEO Paul Maddon. $\mathrm{He}$ adds that Pro140 is "unusual because it binds to the same amino acids [in the receptor protein] as the virus." This binding specificity means that the drug "does not interrupt the functions of this receptor" - which he suggests might be a problem with Pfizer's Selzentry.

Elsewhere, Human Genome Sciences (HGS; Rockville, MD, USA) has used Abgenix's (Fairmont, CA; now part of Amgen) Xenomouse technology to identify several CCR5-directed human mAbs, and initiated a phase 1 trial of CCR5mAb004 in January 2005. Last year, HGS reported that the dose-escalation study carried out on $63 \mathrm{HIV}-1$ positive patients showed that CCR5mAb004 was generally safe and well tolerated, with no severe grade 3 or 4 dose-limiting toxicities.

Taking a different tack, Sangamo BioSciences (Richmond, CA, USA) is developing a biologic that targets the gene encoding CCR5, preventing cells from making this co-receptor protein molecule. The company's zinc-finger DNA-binding endonuclease (ZFN), which is "poised for phase 1" clinical testing, "has very high specificity, precisely cleaving the DNA that we target," says Elizabeth Wolffe, director of communications at Sangamo. If safe, the adenovirus vector-borne product would be administered ex vivo to T cells extracted from HIV-infected individuals, then some treated cells would be reinfused while the remainder, whose "CCR5 gene is permanently disrupted, could provide a reservoir of uninfectable T cells," she says. "This procedure gets around toxicities of small molecules because only $\mathrm{T}$ cells are treated. But it's not something that will be done in a [doctor's] office; it would require clinical centers of excellence."

Apart from HIV co-receptor CCR5, several companies are also focusing on HIV's main receptor, CD4, on T cells and on interfering with the formation of the HIV-cell receptor complex. For example, United Biomedical (Hauppage, NY, USA) is developing a humanized $\mathrm{mAb}(\mathrm{B} 4)$ that is thought to bind to HIV gp120 in complex with the CD4 receptor.

Houston, Texas-based Tanox (now part of Genentech of S. San Francisco, California) had been bullish about its humanized mAb, ibalizumab (TNX-355), which binds CD4 domain 2. Tanox had licensed ibalizumab from BiogenIdec (Cambridge, MA) in 1999 and advanced the product into phase 2 clinical trials by 2003 . Full 48-week data reported in 2006 showed that the $\mathrm{mAb}$ reduced viral load and induced a virologic response in HIV patients, but in August 2006, the FDA advised Tanox that an additional dose-finding clinical trial was needed. Following the completion of Genentech's acquisition of Tanox in August, there has been no news of plans for new trials; however, ibalizumab has been licensed to TaiMed Biologics (Taipei, Taiwan).

Clearly, the potential advantage of targeting host receptors for viruses is that it is extremely difficult for pathogens to develop resistance against the drug. This is because such drugs do not target the virus per se-they target host receptors, which are highly conserved. As Progenics' Maddon puts it: "In the lab, it's almost impossible to develop resistance."

\section{Modulating the host immune system}

Apart from targeting host receptors co-opted by viral pathogens, drug makers also have the opportunity to modulate the host's inherent immune responses to counter infectious agents (Fig. 4). In September, Idera Pharmaceuticals (Cambridge, MA, USA; formerly antisense developer Hybridon) launched a phase 1 trial involving $\mathrm{HCV}$-infected patients to evaluate IMO-2125, an agonist of Toll-like receptor (TLR)-9, a component of the innate immune system. Individuals enrolled in this study, selected from among patients who were failing standard combination therapy of ribavirin and PEG IFN- $\alpha$, are injected with IMO-2125, which is a CpG oligodeoxynucleotide (CpG-ODN)-based molecule designed to stimulate the host immune system through TLR-9, according to Sudhir Agrawal, Idera's CEO and CSO. The secondary structure of IMO-2125 "mimics bacterial DNA," but it is chemically modified to resist degradative enzymes, he says.

Tests in vitro or in animals show that this molecule induces a broad array of immune-system responses, including increases in circulating 


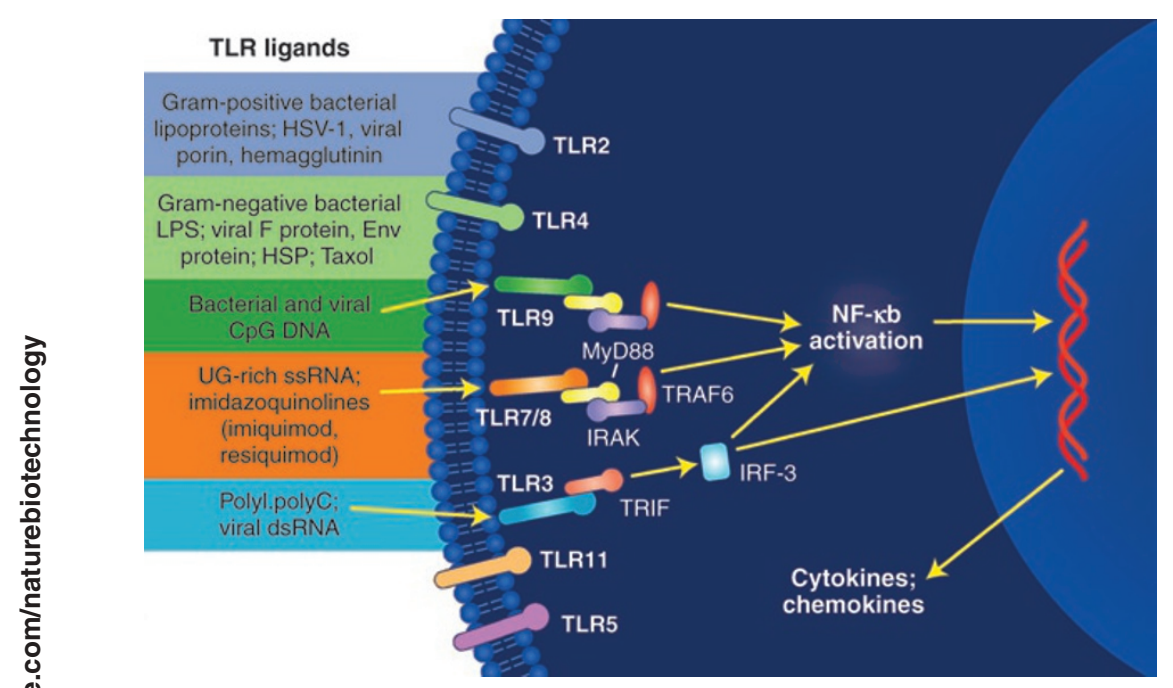

Figure 4 Activation of innate immune system by TRLs. Modulating the host immune response with activators of TRLs is being tested as an approach to controlling viral infections.

IFN- $\alpha$, IFN- $\gamma$ and IL-12, while also activating B cells, T cells and natural killer (NK) cells, 을 Agrawal says. "Once safety and immunological activity are established, we would look to see if we can combine [IMO-2125] with the standard-of-care for HCV patients or even replace IFN- $\alpha$ while still using ribavirin."

Last January, another company, Coley Pharmaceutical (Wellesley, MA, USA) dropped its pursuit of another CpG-ODN TLR-9 agonist, Actilon (CpG-10101), after a phase 2 clinical trial evaluating its activity with standard combination therapy among HCV-infected individuals. After seeing "no meaningful differences in viral load reduction... in any of the treatment arms" during those phase 2 trials, the company suspended further investment in the product. At the time of the announcement, CEO Robert Bratzler said Coley is waiting to "find a partner to share in development costs or... [for] when the path for a second-generation immunomodulating agent becomes clear."

Although Idera's and Coley's TLR-9 agonists are both oligonucleotides, the differences between those two and other immunomodulators "are important for determining the fate of the immune response," says MDB analyst Jasuja. Coley "understood TLR biology...but didn't understand the right way to make a drug, [whereas] Idera's candidate is better constructed to activate TLR-9 in vivo... and is optimized to get release of IL-12." Moreover, he adds, Idera has the expertise to develop stable oligonucleotides to be agonists of the TLR-7 (which recognize single-stranded RNAs; TLR-9 recognizes CpG oligonucleotides).

In another recent set of noteworthy developments involving HBV, Coley granted a license in June to Dynavax Technologies (Berkeley, CA,
USA) to commercialize Heplisav, a combination of a CpG-ODN that stimulates TLR-9 and a HBV surface antigen that invokes both innate and adaptive immune responses. Last month, Dynavax and Merck announced a global license and collaborative agreement to develop Heplisav- touting its "novel TLR 9 agonist" component while also reporting that it confers "immunogenicity after only two doses while retaining tolerability comparable to a currently marketed hepatitis B vaccine." Merck is to pay $\$ 31.5$ million up front to Dynavax, which is eligible to receive $\$ 105$ million in development and sales milestone payments, and double-digit, tiered royalties based on eventual global sales of the product.

In another approach, S. San Francisco, California-based Rigel is developing small molecules that modulate host ubiquitin ligase. This enzyme is hijacked by Vif, an HIV protein required for replication, which recruits a host protein ApoBec39 with antiviral properties into the ubiquitin ligase complex, where it is tagged for destruction. By blocking the ligase, the drug rescues ApoBec39 and HIV replication is repressed.

\section{Other host proteins as targets}

"Viruses take advantage of host molecules, [and] a lot of 'bugs' use the same signaling molecules as the host, particularly tyrosine kinases," says Dan Kalman of Emory University (Atlanta, GA, USA). Thus, he sees opportunities to use drugs that target host-cell molecules and biochemical processes and then "apply them to another purpose." The prototype example is his project examining the antiviral activity of the anticancer drug Gleevec (imatinib mesylate), which is marketed by Novartis Pharmaceuticals (East Hanover, NJ, USA;
Novartis AG, Basel, Switzerland; see Nat. Med. 11, 731-739, 2005).

In brief, Gleevec interferes with Abl-family tyrosine kinases, which are important for the release of poxviruses, such as vaccinia and presumably smallpox, according to Kalman. "It inhibits lots of other viruses, and that's a good thing - a bonanza-from a public health standpoint." Moreover, he adds, "It's wrong to restrict this to virology. If you can develop one drug that hits a lot [of pathogens], it has a bigger market. If one considers anticancer drugs for infectious diseases, what's the limit; why stop at one or two viruses?"

Several other companies are trawling host cell proteins for opportunities to stymie viral replication, maturation or release. Functional Genetics (Gaithersburg, MD, USA) is targeting the cellular protein TSG101 (tumor susceptibility gene) that infecting viruses "hijack and send to cell surfaces abnormally," says Michael Kinch, vice president for R\&D. Apparently, many types of virus depend on this displaced host protein as they "bud out of cells." The company's initial strategy is to identify mAbs that bind TSG101, and one such candidate mAb, FGI-101, by binding to TSG101, interferes with the activity of about two dozen viruses that include Ebola and HIV, according to Kinch. Another candidate product being tested can block both the Ebola and Marburg viruses, providing " $100 \%$ protection prophylactically and post-exposure in vitro and in animals," he says. The approach has "the potential to target biothreat or even unknown viruses.”

In another variation on this theme, NexBio (San Diego, CA) is developing an antiviral approach that entails modification of the host target. Its recombinant protein, Fludase (DAS181), contains one segment with microbially derived sialidase activity that is fused to a cell-surface anchoring segment. The idea is to introduce this protein into airways, allowing it to attach to respiratory epithelium where its catalytic activity would remove sialic acid residues, which serve as receptors for the influenza virus, from host cells. In tests in mice, Fludase treatments improved lung function and survival in animals that were challenged with influenza virus, and the treatments were not toxic to respiratory cells, the company reports.

"The big thing about host-oriented antiviral therapeutics is to change the dogma and the conventional wisdom," says Functional Genetics' Kinch. "Some investigators now see this as the way to go, but it took a lot of slogging to get to this point." More generally, adds Kalman of Emory, "high-throughput is not the way to go. Instead, [study] basic cell biology to find and understand [host] targets, and then find drugs and tune those molecules for safety and specificity." 


\section{Erratum: Antivirals become a broader enterprise}

Jeffrey L Fox

Nat. Biotechnol. 25, 1395-1402 (2007); published 7 December 2007; corrected after print 8 April 2008

In the version of this article initially published, the name of a person quoted on p. 1399 was incorrect. The CSO of Replicor, Laval, QC, Canada, is Andrew Vaillant, not "Andrew Toussaint" as originally published. The error has been corrected in the HTML and PDF versions of the article. 